Apidologie, 1984, 15 (2), 145-170

\title{
LES MIELS D'AFRIQUE DU NORD ET LEUR SPECTRE POLLINIQUE
}

\author{
J. LOUVEAUX * et L. ABED ** \\ * I.N.R.A. Station de recherches sur l'abeille et les insectes sociaix \\ F 91440 Bures-sur-Yvette \\ * Laboratoire de Matière Médicale, Institut de Pharmacie, Alger, Algérie \\ et U.E.R. de Pharmacie, 1, rue Vaubenard, F-14032 Caen Cedex
}

\begin{abstract}
RÉSUMÉ
Le spectre pollinique des miels d'Afrique du Nord est relativement mal connu. C'est pourquoi il a semblé utile, pour améliorer nos connaissances à ce sujet, de rassembler les résultats de 87 analyses polliniques (Maroc 16, Algérie 59, Tunisie 12) réalisées à la Station de Bures-sur-Yvette entre 1949 et 1982. Ces résultats sont regroupés en 4 tableaux concernant les miels dont le spectre pollinique comporte un pollen dominant (Eucalyptus, Citrus, Rosmarinus,....) et un tableau concernant les miels dont le spectre pollinique ne comporte pas de pollen dominant. La discussion porte sur l'importance relative des plantes mellifères du Maghreb et sur l'intérêt de poursuivre les recherches compte tenu du nombre assez élevé de taxons identifiés à un niveau de précision insuffisant.
\end{abstract}

\section{INTRODUCTION}

La mélisso-palynologie ou palynologie appliquée à l'apidologie contribue étroitement à la connaissance des rapports de tous ordres qui existent entre l'abeille et la plante. A ce titre elle s'intègre aux recherches écologiques, éthologiques et physiologiques car elle permet l'utilisation du grain de pollen comme marqueur et indicateur biologique dans le vaste contexte des relations plante-abeille.

L'analyse pollinique des miels est la plus connue mais non l'unique branche de la mélisso-palynologie. C'est la plus ancienne puisqu'elle a ses origines dans les observations de Pfister (1895) sur la présence constante de grains de 
pollen dans les miels. Elle s'est considérablement développée en Allemagne avec les travaux de ZanDER (de 1935 à 1951) et en Suisse depuis 1936 avec ceux de MAURIZIO.

La bibliographie de l'analyse pollinique des miels est à la fois abondante et dispersée. On en trouve l'essentiel pour la période 1895-1975 dans les ouvrages. généraux (A. Maurizio et J. Louveaux, 1965 ; J. Louveaux, 1968 ; A. MauRIZIO, 1975). On peut classer l'ensemble des publications relatives à l'analyse pollinique des miels dans trois catégories : méthodologie, monographies régionales, études ponctuelles.

La méthodologie de l'analyse pollinique des miels s'éloigne sensiblement de celles de la palynologie générale car elle répond à des besoins particuliers. On en trouve l'exposé le plus récent dans l'article de J. Louveaux, A. Maurizio et G. VORWOHL, 1978.

Les monographies régionales prennent pour cadre un pays ou une grande région géographique. On peut citer comme exemple caractéristique de ces monographies le travail de F. RutTnER (1956) sur les miels de la Haute Autriche ou, beaucoup plus récente, celle de M.-J. Feller-Demalsy (1983) sur les micis du Québec. Ces grandes études, lorsqu'elles sont conduites selon des méthodes. appropriées, améliorent nos connaissances sur la flore mellifère du monde ct sur les ressources alimentaires dont disposent les abeilles en fonction des facteurs écologiques essentiels. Ces connaissances progressent d'année en année.

Les études ponctuelles ne concernent qu'un domaine géographique limité ou un type de milieu précis. Elles peuvent également ne concerner qu'une plante ou un groupe de plantes mellifères. Malgré leur caractère ponctuel ces études peuvent revêtir une grande importance lorsqu'elles apportent une information décisive. Ainsi, en 1960, A. Maurizio a réussi à identifier de façon certaine le pollen de Loranthus europaeus dans certains miels d'Europe centrale. Cette découverte, confirmée par F. RutTner $(1961,1964)$ a permis de résoudre le problème délicat de l'identification de miels d'acacia (Robinia pseudacacia) du Bassin danubien. Une étude complémentaire (A. Fossel et F. RutTNER, 1966) portant sur une Borraginacée d'Europe centrale, Cerinthe minor a bien contribué à caractériser les miels de la plaine parnonique.

Si l'on fait le bilan des études régionales publiées depuis un demi-siècle environ on se rend compte qu'il existe encore un décalage important entre la connaissance que nous avons des miels européens et celle des miels des autres parties du monde. Ainsi, les miels en provenance de l'Ouest du Bassin méditerranéen et en particulier de l'Afrique du Nord restent relativement mal connus du point de vue de leur spectre pollinique. Ils n'ont pas fait jusqu'ici l'objet d'une 
étude exhaustive. Les seules publications dont nous disposions actuellement sont celles de Vorwohl $(1973,1981)$ sur les miels de Tunisie et celle de Ricciardelli D'Albore sur les miels du Maroc (1980). Quelques informations fragmentaires se trouvent dispersées dans diverses publications dont l'objet n'est pas directement lié aux miels d'Afrique du Nord.

Cette situation est d'autant plus regrettable que l'Afrique du Nord représente une aire de production apicole potentiellement importante du fait de sa flore mellifère abondante et variée et de son climat généralement très favorable aux abeilles (SKender, 1972 ; Paradeau, 1978). La présente étude qui ne prétend pas être exhaustive a pour simple objet de présenter un ensemble de résultats d'analyses polliniques restés jusqu'ici inédits mais dont la publication peut motiver ultérieurement des recherches approfondies.

\section{MATERIEL ET METHODES}

Au fil des années, la Station de Recherches sur l'Abeille et les Insectes sociaux a recueilli un certain nombre d'échantillons de miel en provenance d'Afrique du Nord. Généralement ces échantillons nous ont été adressés en vue d'une analyse pollinique informative par les producteurs eux-mêmes ou par l'intermédiaire d'un apiculteur désireux de contribuer à une étude générale des miels africains. Il ne s'agit donc pas d'échantillons provenant du commerce; leur origine géographique est connue, soit de façon très précise, soit de façon plus large mais, en tout état de cause, nous n'avons conservé pour cette étude que les miels dont l'origine nous était garantie.

Dans la période la plus récente nous avons bénéficié de la collaboration directe d'apiculteurs maghrébins par l'intermédiaire desquels nous avons pu disposer d'échantillons représentatifs de la production actuelle.

Le tableau 1 donne la liste des échantillons analysés. L'année d'enregistrement se confond le plus souvent avec l'année de production qui peut cependant parfois être une année antérieure.

En raison de la longueur de la période étudiée (32 ans) et du fait que certains échantillons ne sont plus en collection pour des raisons diverses, la présentation des résultats n'est pas homogène. Nous nous sommes efforcés d'atténuer le plus possible ces différences de présentation sans toutefois pouvoir les supprimer complètement.

Toutes les analyses polliniques ont été faites selon les méthodes classiques de la mélisso-palynologie (J. LouveauX, A. Maurizio et G. Vorwohl, 1978). Les analyses effectuées avant 1978 l'ont été selon des méthodes très peu différentes, de telle sorte que l'on peut admettre, dans l'optique de cette étude principalement qualitative, que les résultats sont comparables. 
TABL. 1. - Présentation des échantillons

TABL. 1. - Survey of samples

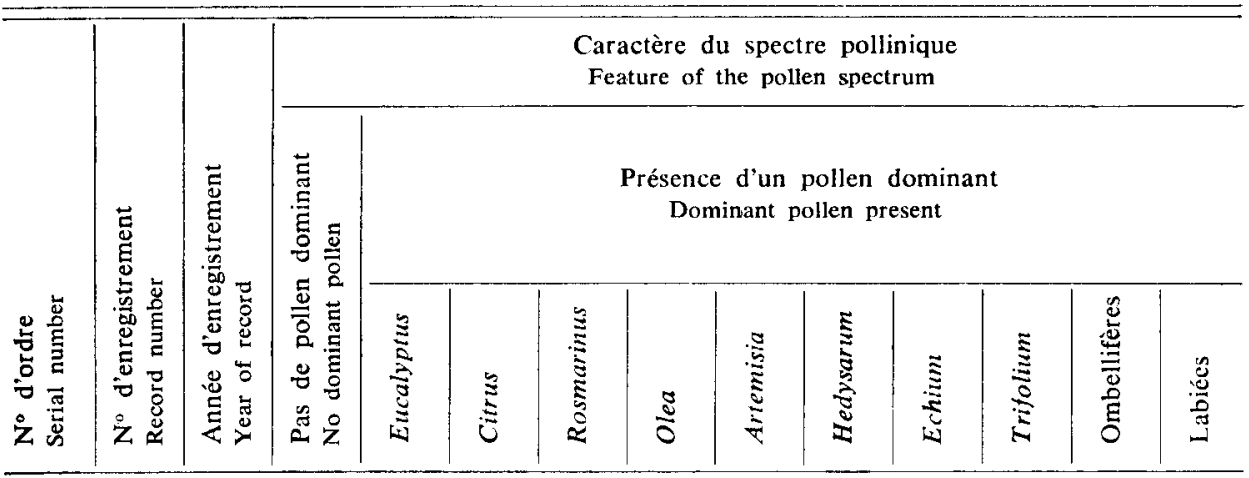

ALGERIE : Oranie

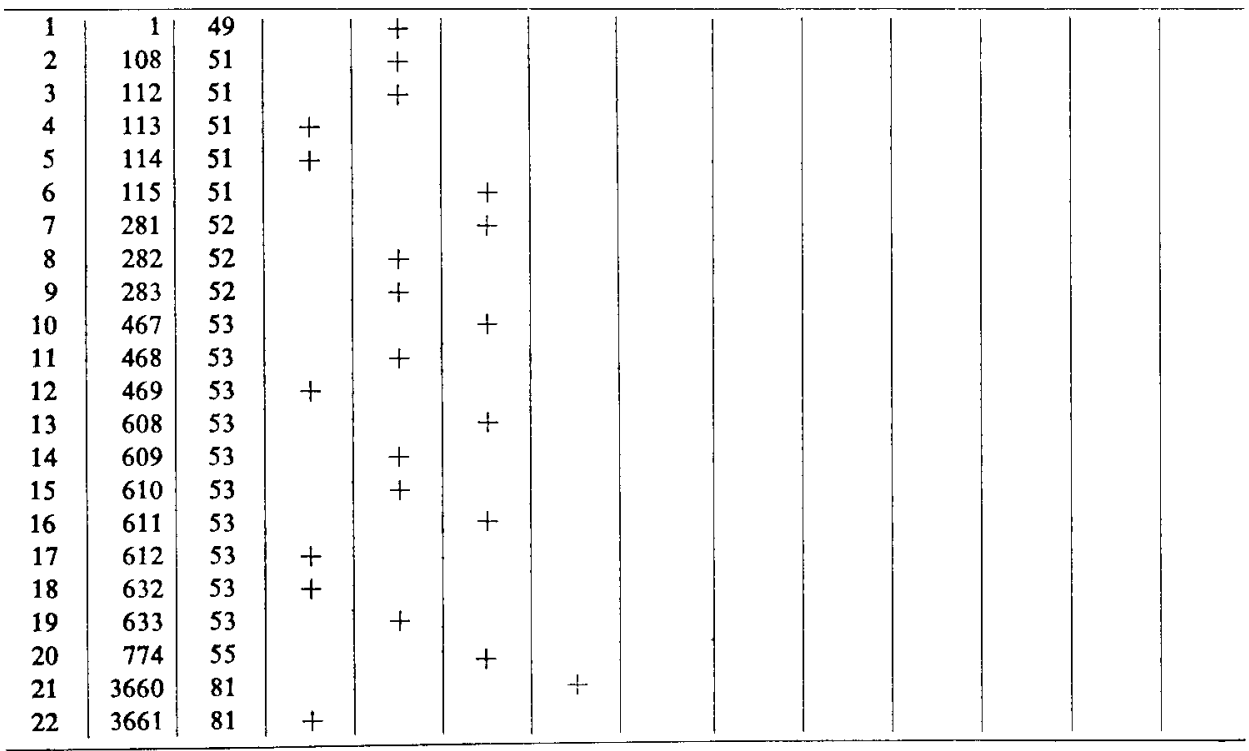

ALGERIE : Algérois

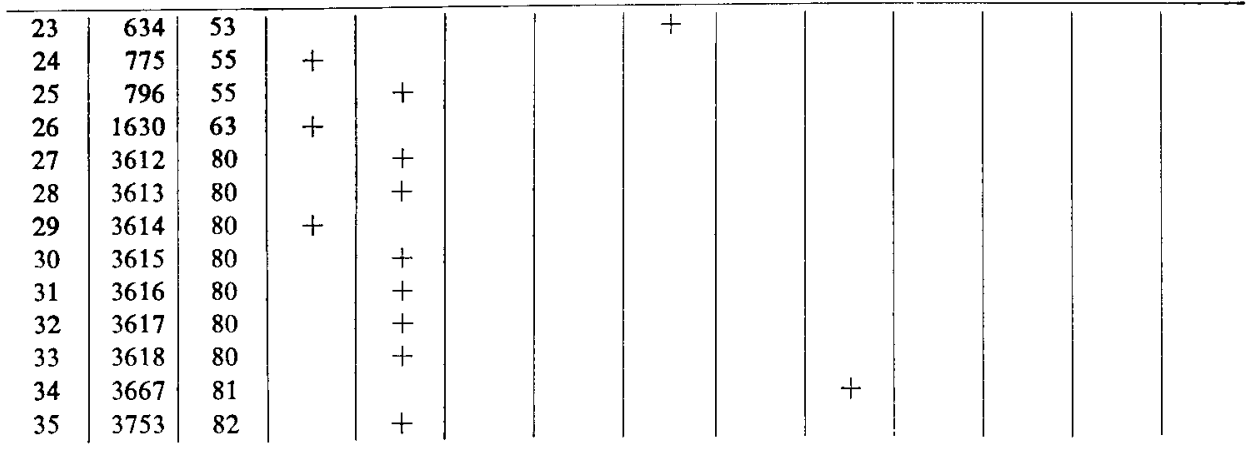


TABL. 1. - (suite)

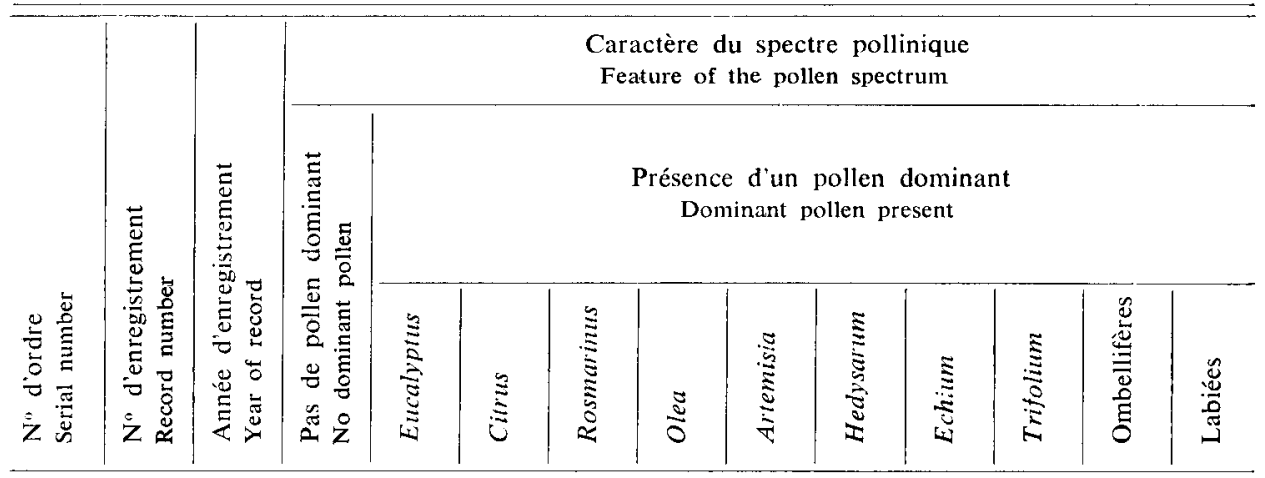

ALGERIE : Constantinois

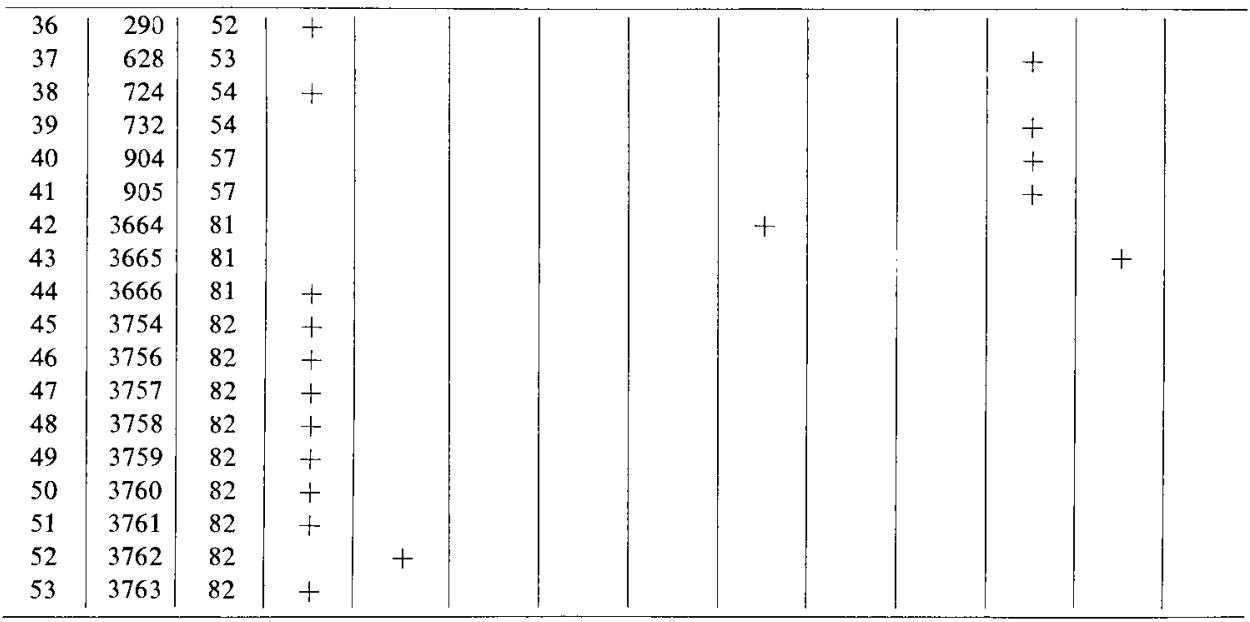

ALGERIE : $S_{\text {ud-Algérien }}$

\begin{tabular}{l|l|l|l|l|l|l|l|}
\hline 54 & 3662 & 81 & + & & & & \\
55 & 3663 & 81 & + & & & & \\
56 & 3755 & 82 & & & & & \\
\hline
\end{tabular}

Divers

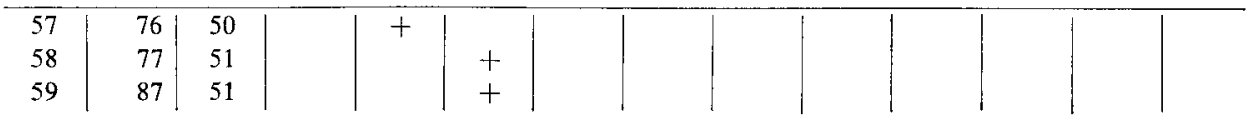


TABL. 1. - (suite)

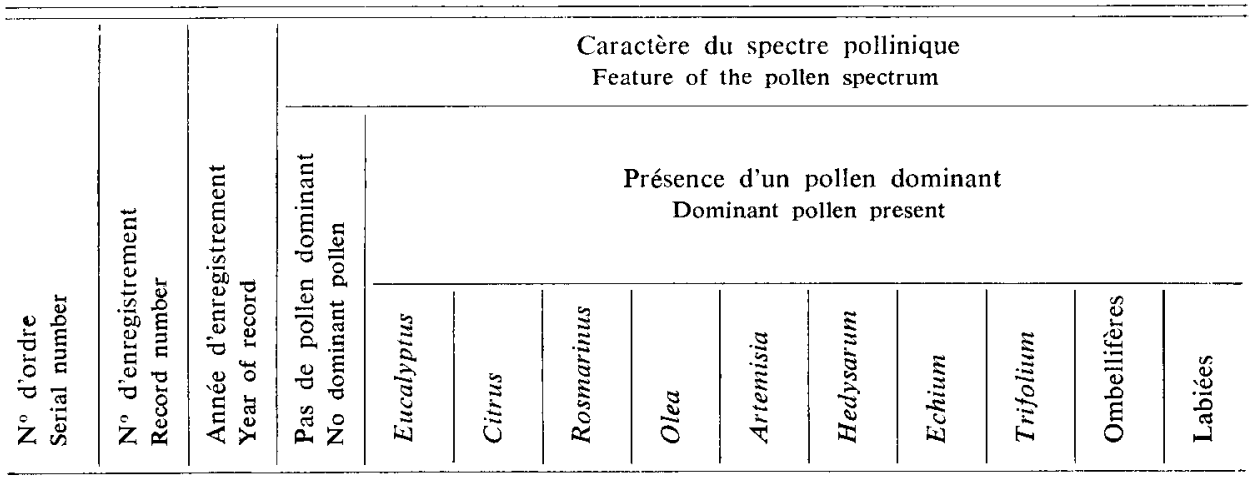

MAROC : Rharb

\begin{tabular}{l|r|r|r|r|r|}
60 & 103 & 51 & & & \\
61 & 291 & 52 & & & \\
62 & 2314 & 64 & & + & \\
63 & 2915 & 66 & & + & \\
64 & 3671 & 81 & & + &
\end{tabular}

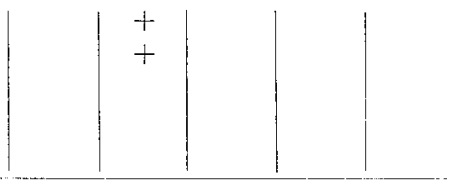

\section{Divers}

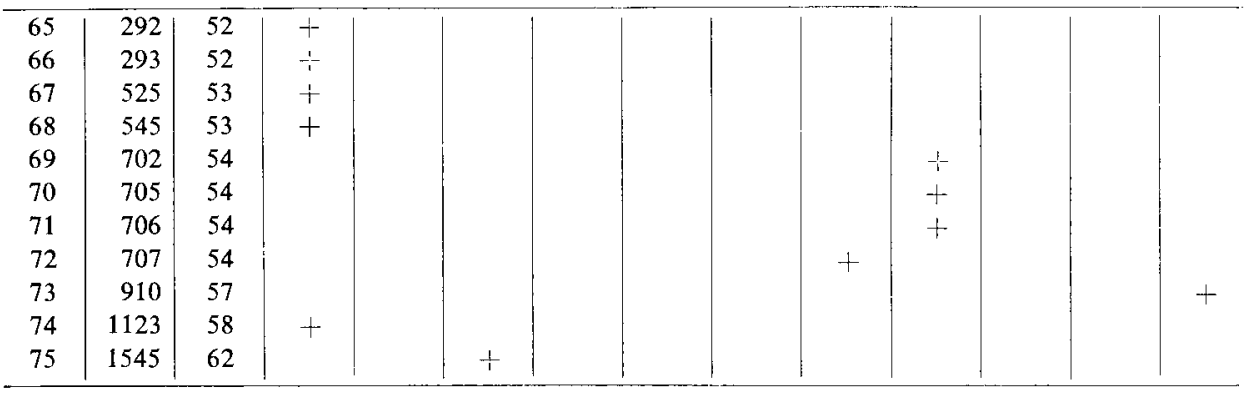

\section{TUNISIE}

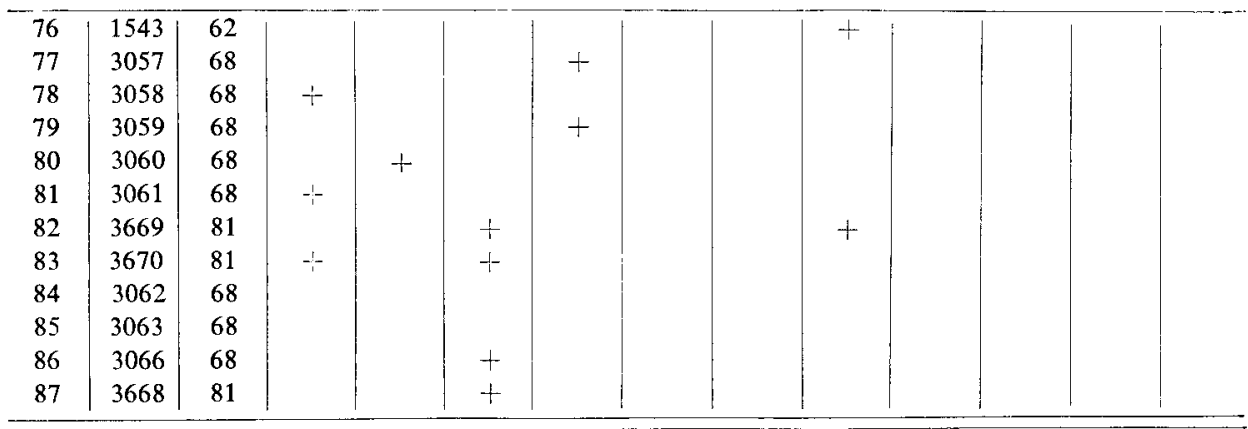




\section{RÉSULTATS}

Le tableau 1 qui constitue une liste complète des 87 échantillons étudiés comporte un classement double indépendamment du classement chronologique. Nous avons regroupé les échantillons en fonction de leur origine géographique d'une part, en fonction de leur spectre pollinique d'autre part. En Algérie et au Maroc nous avons introduit des subdivisions géographiques (Fig. 1) ce que nous n'avons pas fait en Tunisie où les échantillons proviennent tous de la partie nord du pays. En ce qui concerne le classement en fonction de l'origine botanique nous avons pris en considération le spectre pollinique brut chiffré ou estimé (Louveaux, 1968). Cette solution imparfaite nous est apparue comme la seule possible ; en effet, certains miels obtenus par pressage des rayons présentent une richesse de pollen qui rend trop difficile une interprétation correcte de leur origine florale.

\section{Miels dont le spectre pollinique comporte un pollen dominant}

\subsection{Miels d'Eucalyptus (Tabl. 2)}

Les miels d'Eucalyptus sont, en règle générale, riches en pollen. Il n'est donc pas étonnant que certains échantillons fassent apparaître des pourcentages de pollen d'Eucalyptus approchant de $100 \%$. Nous avons volontairement renoncé à indiquer des noms d'espèces à l'intérieur du genre Eucalyptus étant donné d'une part qu'il s'agit de pollens morphologiquement très voisins et que, d'autre part, les auteurs préparent une étude plus détaillée du spectre pollinique des miels d'Eucalyptus de la région d'Alger.

On remarque que les miels d'Eucalyptus sont bien représentés sur l'ensemble de l'Afrique du Nord et qu'on les trouve aussi bien au début qu'à la fin de la période étudiée (1949-1982). Ceci nous permet de conclure à l'importance des Eucalyptus en tant que plantes mellifères maghrébines, ce qui était certes connu mais que nous pouvons confirmer de façon particulièrement nette.

D'une façon générale la flore d'accompagnement présente dans les miels d'Eucalyptus est assez homogène. Les pollens de Citrus, Olea, Echium, Chamaerops, Ombellifères diverses, sont le plus souvent présents dans les spectres considérés.

\subsection{Miels de Citrus (Tabl. 3)}

Sous le nom générique de Citrus nous comprenons les diverses Aurantiacées cultivées (Oranger, Citronnier, Mandarinier, ...). Cependant l'usage du terme «miel d'oranger» dans le grand public est largement justifié par le fait que les miels de Citrus aurantium représentent l'essentiel de la production. 


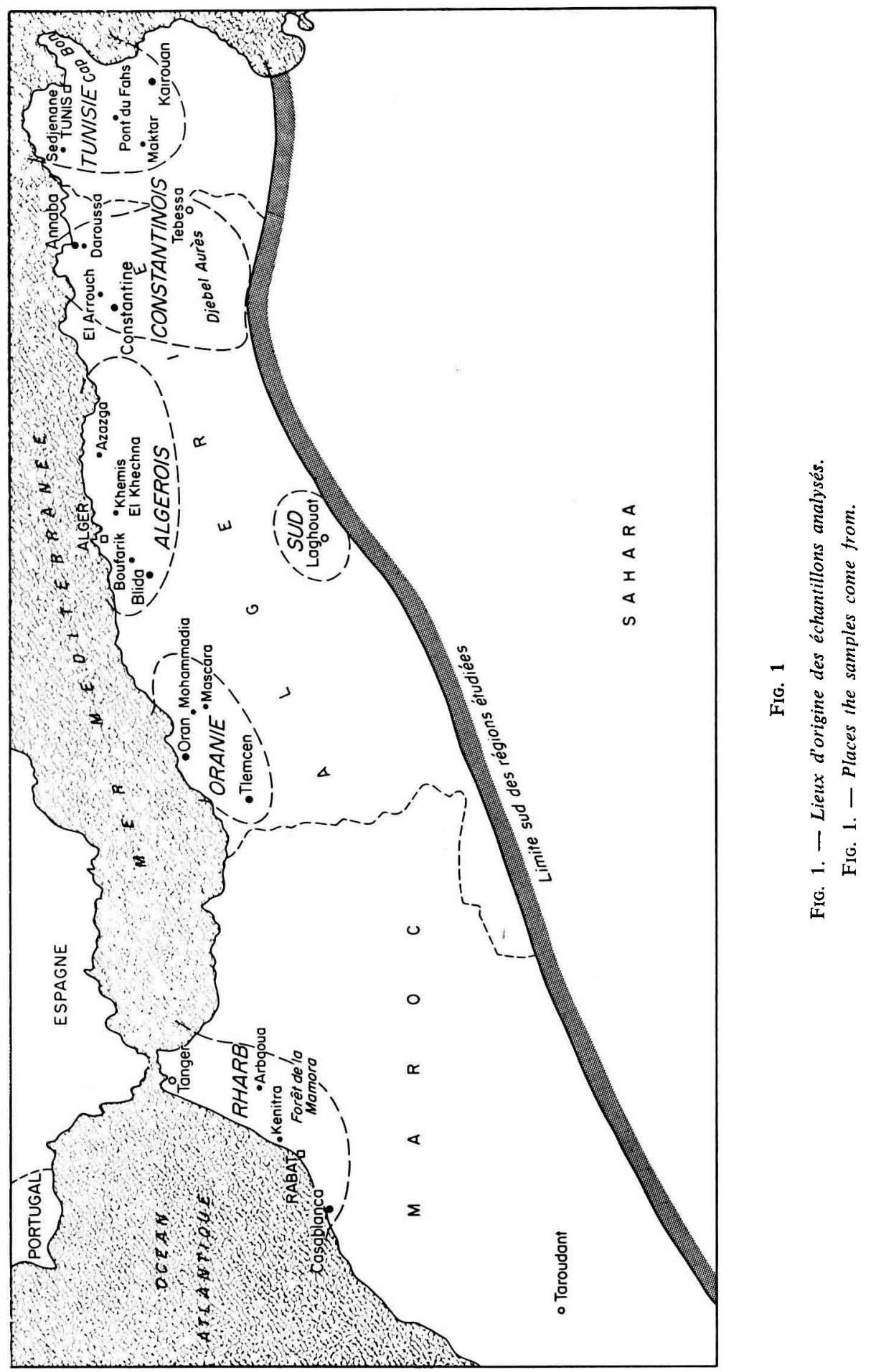


TAвl. 2. - Pollen d'Eucalyptus dominant

TABL. 2. - Dominant Eucalyptus pollen

\begin{tabular}{|c|c|c|c|c|}
\hline 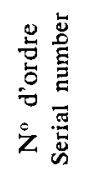 & 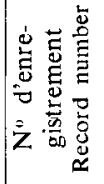 & 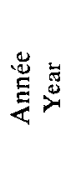 & $\begin{array}{l}\text { Origine } \\
\text { des échantillons } \\
\text { Origin of samples }\end{array}$ & $\begin{array}{c}\text { Spectre pollinique - Observations } \\
\text { Pollen spectrum - Observations }\end{array}$ \\
\hline
\end{tabular}

ALGERIE : Oranie

\begin{tabular}{|c|c|c|c|c|}
\hline 1 & 1 & 49 & $\begin{array}{l}\text { Mohammadia } \\
\text { (ex Perrégaux) }\end{array}$ & $\begin{array}{l}\text { Miel présumé d'Eucalyptus - Odeur et saveur Euca- } \\
\text { lyptus. } \\
\text { Eucalyptus } \simeq 100 \text { - Divers, } 1 \text { : Citrus - Acacia - } \\
\text { Crucifères - Composśes type Taraxacum. Spores - } \\
\text { Levures - Corps étrangers indéterminés - Miellat } \\
\text { possible. }\end{array}$ \\
\hline 2 & 108 & 51 & Mohammadia & $\begin{array}{l}\text { Miel piésumé d'Eucalyptus rostrata. } \\
\text { Eucalyptus, } 96 \text { - Divers, } 4 \text { : Citrus - Olea - Om- } \\
\text { bellifères. }\end{array}$ \\
\hline 3 & 112 & 51 & Mohammadia & $\begin{array}{l}\text { Miel présumé de Coton et Inule visqueuse. } \\
\text { Eucalyptus, } 58 \text { - Olea, } 15 \text { - Citrus, } 2 \text { - Crucifères, } 3 \text { - } \\
\text { Ombellifères, } 5 \text { - Cistus, 1 - Inula, 1 - Echitum, } 1 \text { - } \\
\text { Divers et indéterminés, 14. }\end{array}$ \\
\hline 8 & 282 & 52 & $\begin{array}{c}\text { Oranie } \\
\text { (sans autre précision) }\end{array}$ & $\begin{array}{l}\text { Saveur Eucalyptus. } \\
\text { Eucalyptus, } 52 \text { - Citrus, } 9 \text { - Olea, } 2 \text { - Caryophyllacées, } \\
4 \text { - Cistus, } 1 \text { - Ericacée, } 2 \text { - Echium, } 2 \text { - Crucifères, } \\
2 \text { - Graminées, } 2 \text { - Papilionacées, } 1 \text { - Rosacśes, } 1 \text { - } \\
\text { Divers et indéterminés, } 22 \text { - Miellat probable. } \\
\text { Spores nombreuses - Algues vertes - Levures - Déb:is } \\
\text { d'insectes - Grains d'amidlon. }\end{array}$ \\
\hline 9 & 283 & 52 & $\begin{array}{c}\text { Oranie } \\
\text { (sans autre précision) }\end{array}$ & $\begin{array}{l}\text { Saveur Citrus faible. } \\
\text { Eucalyptus, } 47 \text { - Citrus, } 22 \text { - Olea, } 4 \text { - Cistus - Ca- } \\
\text { ryophyllacées, } 2 \text { - Salix, } 4 \text { - Rumex, } 1 \text { - Acacia, } 1 \text { - } \\
\text { Papilionacées, } 3 \text { - Crucifères, } 2 \text { - Divers et indéter- } \\
\text { minés, } 10 .\end{array}$ \\
\hline 11 & 468 & 53 & Mohammadia & $\begin{array}{l}\text { Miel présumé d'Eucalyptus. } \\
\text { Eucalyptıs, } 98 \text { - Citrus, } 1 \text { - Ombellifères } 1 . \\
\text { Présence de levures et corps étrangers indéterminés. }\end{array}$ \\
\hline 14 & 609 & 53 & Mohammadia & $\begin{array}{l}\text { Miel présumé d'Eucalyptus. } \\
\text { Eucalyptus, } 97 \text { - Divers, } 3 \text { : Citrus - Mimosacées - } \\
\text { Ombellifères - Crucifères. }\end{array}$ \\
\hline 15 & 610 & 53 & Mohammadia & $\begin{array}{l}\text { Miel présumé d'Eucalyptus. } \\
\text { Eucalyptus (formes diverses), } 97 \text { - Divers, } 3 \text { : Cirrus - } \\
\text { Olea - Crucifères - Ombellifères. }\end{array}$ \\
\hline 19 & 633 & 53 & Mohammadia & $\begin{array}{l}\text { Miel présumé d'Eucalyptıs. } \\
\text { Eucalyptus, } 96 \text { - Divers } 4: \text { : Caryophyllacées - Cen- } \\
\text { taurea - Ombellifères. }\end{array}$ \\
\hline
\end{tabular}


TABL. 2. 一 (suite)

\begin{tabular}{|c|c|c|c|c|}
\hline 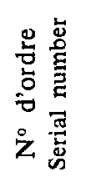 & 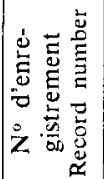 & $\stackrel{\mathscr{E}}{\Xi}$ & $\begin{array}{l}\text { Origine } \\
\text { des échantillons } \\
\text { Origin of samples }\end{array}$ & $\begin{array}{l}\text { Spectre pollinique - Observations } \\
\text { Pollen spectrum - Observations }\end{array}$ \\
\hline
\end{tabular}

Algérois

\begin{tabular}{|c|c|c|c|c|}
\hline 25 & 796 & 55 & $\begin{array}{c}\text { Khemis el Khechna } \\
\text { (Ex. Fondouk) }\end{array}$ & Eucalyputs $=100$. \\
\hline 27 & 3612 & 80 & Alger-Hydra & $\begin{array}{l}\text { Miel récolté en août } 1979 . \\
\text { Eucalyptus }=100 \text { - Divers : Quercus-Olea-Ombel- } \\
\text { lifères. }\end{array}$ \\
\hline 28 & $36 ! 3$ & 80 & Alger-Hydra & $\begin{array}{l}\text { Miel récolté en juillet } 1978 . \\
\text { Eucalyptus } \simeq 100-\text { Divers : Trifolium - Lotus - } \\
\text { Hedysarum - Olea - Chamaerops. }\end{array}$ \\
\hline 30 & 3615 & 80 & Alger-Hydra & $\begin{array}{l}\text { Miel récolté en octobre } 1980 \text { - (Seconde récolte). } \\
\text { Eucalyptus, } 87 \text { - Divers, } 13 \text { : Papilionacées - Quer- } \\
\text { cus - Composées diverses - Ombellifères - Cha- } \\
\text { maerops. }\end{array}$ \\
\hline 31 & 3616 & 80 & Alger-Hydra & $\begin{array}{l}\text { Miel récolté en août } 1976 \text {. } \\
\text { Eucalyptus } \simeq 100 \text { - Divers : Olea, Malvacées, Papi- } \\
\text { lionacée. }\end{array}$ \\
\hline 32 & 3617 & 80 & Alger-Hydra & $\begin{array}{l}\text { Miel récolté en août } 1977 . \\
\text { Eucalyptus, } 88 \text { - Divers, } 12 \text { : Trifolium, Lotus, } \\
\text { Hedysarum, Olea, Chamaerops. }\end{array}$ \\
\hline 33 & 3618 & 80 & Alger-Hydra & $\begin{array}{l}\text { Miel récolté en août } 1975 \text {. } \\
\text { Eucalyptus }=100-\text { (Rares pollens isolés type Pal- } \\
\text { mier). } \\
\text { Nombreuses spores - Miellat possible. }\end{array}$ \\
\hline 35 & 3753 & 82 & Alger-Hydra & $\begin{array}{l}\text { Miel récolté en août } 1981 \text { - Prélèvement sur un } \\
\text { rayon - récolte par égouttage. } \\
\text { Eucalyptus } \simeq 100 \text { (plusieurs espèces). } \\
\text { Quelques grains d'amidon. }\end{array}$ \\
\hline
\end{tabular}

Constantinois

\begin{tabular}{l|l|l|l|l}
\hline 52 & 3762 & 82 & $\begin{array}{c}\text { Constantinois } \\
\text { (sans autre précision) }\end{array}$ & $\begin{array}{l}\text { Miel présumé d'Eucalyptus. } \\
\text { Eucalyptus }=100-\text { Divers : Hedysarum - Cistus - } \\
\text { Crucifères. } \\
\text { Parmi les pollens d'Eucalyptus un type est fortement } \\
\text { dominant. }\end{array}$ \\
\hline
\end{tabular}

Sud-Algérien

\begin{tabular}{|c|c|c|c|c|}
\hline 56 & 3755 & 82 & Laghouat & $\begin{array}{l}\text { Miel récolté en été } 1979-\text { Miel d'extracteur. } \\
\text { Eucalyptus } \simeq 100-\text { (Plusieurs types) - Divers : } \\
\text { Rumex - Crucifères. } \\
\text { Sédiment riche en particules minérales (sable?), } \\
\text { suie, fibres, spores, fragments de propolis, levures. }\end{array}$ \\
\hline
\end{tabular}


TABL. 2. - (suite)

\begin{tabular}{|c|c|c|c|c|}
\hline 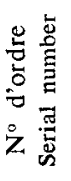 & 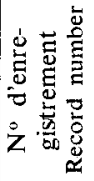 & 总 & $\begin{array}{l}\text { Origine } \\
\text { des échantillons } \\
\text { Origin of samples }\end{array}$ & $\begin{array}{c}\text { Spectre pollinique - Observations } \\
\text { Pollen spectrum - Observations }\end{array}$ \\
\hline
\end{tabular}

Divers

\begin{tabular}{c|c|c|c|c|c}
\hline 57 & 76 & 50 & $\begin{array}{c}\text { Algérie } \\
\text { (sans autre précision) }\end{array}$ & $\begin{array}{l}\text { Eucalyptus, 81 - Papilionacées type Trifolium, 10 - } \\
\text { Ombellifères, 1 - Divers et indéterminés, 8. }\end{array}$ \\
\hline
\end{tabular}

MAROC : Rharb

\begin{tabular}{|c|c|c|c|c|}
\hline 62 & 2314 & 64 & Rharb-Arbaoua & $\begin{array}{l}\text { Miel présumé d'Eucalyptus. } \\
\text { Eucalyptus, } 95 \text { - (Plusieurs types) - Pollens isolés : } \\
\text { Acacia - Trifolium - Crucifères - Forte proportion } \\
\text { de grains de pollen d'Eucalyptus avortés. } \\
\text { Sédiment riche en particules étrangères - Nombreuses } \\
\text { spores. }\end{array}$ \\
\hline 63 & 2915 & 66 & Rharb & $\begin{array}{l}\text { Miel présumé d'Eucalyptus - Rucher situé en forêt } \\
\text { d'Eucalyptus - Récolté en juillet } 1966 \text {. } \\
\text { Eucalyptus, } 98 \text { - Pas de pollens isolés identifiables - } \\
\text { Forte proportion de grains de pollen d'Eucalyptus } \\
\text { avortés. } \\
\text { Présence de levures. }\end{array}$ \\
\hline 64 & 3671 & 81 & $\begin{array}{l}\text { Forêt de la Mamora } \\
\text { Kenitra } \\
\text { (Ex. Port-Lyautey) }\end{array}$ & $\begin{array}{l}\text { Miel présumé d'Eucalyptus. } \\
\text { Eucalyptus, } 98 \text { - Divers : Composées diverses } \\
\text { Cistus - Plantago - Trifolium - Acacia. }\end{array}$ \\
\hline
\end{tabular}

\section{TUNISIE}

\begin{tabular}{|c|c|c|c|c|}
\hline 80 & 3060 & 68 & Tunis-La Soukra & $\begin{array}{l}\text { Miel présumé d'Eucalyptus - Récolté en octobre } \\
1967 \text { après première récolte sur oranger en juin } 1967 \text { - } \\
\text { Miel d'extracteur. } \\
\text { Eucalyptus, } 80 \text { - Divers, } 20 \text { : Olea - Citrus - Echium - } \\
\text { Diplotaxis - Rosmarinus - Salix - Hedysarum - } \\
\text { Composées diverses - Liliacées - Très nombreuses } \\
\text { spores - Présence probable de miellat. }\end{array}$ \\
\hline
\end{tabular}

Le tableau 3 montre bien le caractère régional de la production des miels de Citrus. On les trouve essentiellement dans les zones de cultures d'agrumes : Ouest algérien, Nord tunisien. 
Tabl. 3. - Pollen de Citrus dominant

TaBL. 3. - Dominant Citrus pollen

\begin{tabular}{|c|c|c|c|c|}
\hline 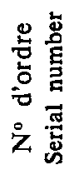 & 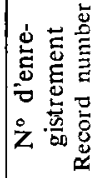 & 䕇芯 & $\begin{array}{l}\text { Origine } \\
\text { des échantillons } \\
\text { Origin of samples }\end{array}$ & $\begin{array}{c}\text { Spectre pollinique - Observations } \\
\text { Pollen spectrum - Observations }\end{array}$ \\
\hline
\end{tabular}

ALGERIE : Oranie

\begin{tabular}{|c|c|c|c|c|}
\hline 6 & 115 & 51 & Mohammadia & $\begin{array}{l}\text { Miel présumé d'oranger. } \\
\text { Citrus, } 51 \text { - Olea, } 18 \text { - Eucalyptus, } 11 \text { - Crucifères, } \\
10 \text { - Cistus, } 2 \text { - Inula, } 1 \text { - Ombellifères, } 1 \text { - Divers et } \\
\text { indéterminés, } 6 \text {. }\end{array}$ \\
\hline 7 & 281 & 52 & Mohammadia & $\begin{array}{l}\text { Miel présumé d'oranger - Saveur de miel d'oranger - } \\
\text { Pauvre en pollen - Citrus, } 36 \text { - Caryophyllacées, } 17 \text { - } \\
\text { Cistus, } 7 \text { - Salix, } 1 \text { - Oled, } 1 \text { - Rumelx, } 1 \text { - Chénopo- } \\
\text { diacées, } 1 \text { - Arbres fruitiers, } 1 \text { - Papilionacées, } 4 \text { - } \\
\text { Acacia, } 1 \text { - Echium, } 11 \text { - Plantago, } 1 \text { - Graminées, } 1 \text { - } \\
\text { Divers et indéterminés, } 17 \text {. } \\
\text { Nombreuses spores - Algues vertes - Débris divers - } \\
\text { Levures - Miellat probable. }\end{array}$ \\
\hline 10 & 467 & 53 & Mohammadia & $\begin{array}{l}\text { Miel présumé d’oranger - Saveur de miel d’oranger - } \\
\text { Pauvre en pollen. } \\
\text { Citrus, } 53 \text { - Olea, } 16 \text { - Euralyptus, } 20 \text { - Vicia, } 1 \text { - } \\
\text { Ombellifères, } 3 \text { - Crucifères, } 3 \text { - Divers et indéter- } \\
\text { minés, } 4 . \\
\text { Présence de grains d'amidon - Spores - Levures. }\end{array}$ \\
\hline 13 & 608 & 53 & Mohammadia & $\begin{array}{l}\text { Miel présumé d'Oranger. } \\
\text { Citrus, } 45 \text { - Eucalyptus, } 23 \text { - Olea, } 22 \text { - Cynoglossum, } \\
4 \text { - Crucifères, } 4 \text { - Lotus, } 1 \text { - Divers, } 1 .\end{array}$ \\
\hline 16 & 611 & 53 & Mohammadia & $\begin{array}{l}\text { Miel présumé d'oranger. } \\
\text { Citrus, } 39 \text { - Olea, } 13 \text { - Lotus, } 15 \text { - Echium, } 9 \text { - } \\
\text { Crucifères, } 7 \text { - Acacia, } 1 \text { - Divers, } 16 \text { [dont Juni- } \\
\text { perus (?) - Plantago - Ombellifères). }\end{array}$ \\
\hline 20 & 774 & 55 & Mohammadia & $\begin{array}{l}\text { Miel présumé d’oranger. } \\
\text { Citrus - Olea - Eucalyptus - Pollens isolés : Lavan- } \\
\text { dula - Cerinthe - Crucifères - Trifolium - Ombelli- } \\
\text { fères - Echium. }\end{array}$ \\
\hline
\end{tabular}

Divers

\begin{tabular}{|c|c|c|c|c|}
\hline 58 & 77 & 51 & $\begin{array}{l}\text { Algérie } \\
\text { (sans autre précision) } \\
\text { mais probablement } \\
\text { originaire d'Oranie }\end{array}$ & $\begin{array}{l}\text { Miel présumé d'oranger - Siaveur de miel d'oranger - } \\
\text { Pauvre en pollen. } \\
\text { Citrus, } 31 \text { - Olea, } 34 \text { - Chénopodiacées, } 7 \text { - Salix, } 8 \text { - } \\
\text { Crucifères, } 2 \text { - Eucalyptus, } 2 \text { - Trifolium, } 1 \text { - Caryo- } \\
\text { phyllacécs, } 2 \text { - Composées, } 1 \text { - Divers et indéter- } \\
\text { minés, } 12 \text {. } \\
\text { Présence de grains d'amidon, spores, algues vertes, } \\
\text { corps étrangers inorganiques - Présence de miellat } \\
\text { possible. }\end{array}$ \\
\hline
\end{tabular}


TABL. 3. - (suite)

\begin{tabular}{|c|c|c|c|c|}
\hline 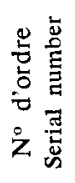 & 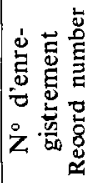 & 兽 & $\begin{array}{l}\text { Origine } \\
\text { des échantillons } \\
\text { Origin of samples }\end{array}$ & $\begin{array}{c}\text { Spectre pollinique - Observations } \\
\text { Pollen spectrum - Observations }\end{array}$ \\
\hline
\end{tabular}

\begin{tabular}{c|c|c|c|c}
\hline 59 & 57 & $\begin{array}{c}\text { Même remarque } \\
\text { que pour l'échantillon } \\
\text { précédent }\end{array}$ & $\begin{array}{l}\text { Miel présumé d'oranger. } \\
\text { Citrus, 36-Papilionacées type Trifolium, 32-Euca- } \\
\text { lyptus, 4-Crucifères, 4. - Divers et indéterminés, 24. }\end{array}$ \\
\hline
\end{tabular}

\section{MAROC}

\begin{tabular}{|c|c|c|c|c|}
\hline 75 & 1545 & 62 & $\begin{array}{c}\text { Maroc } \\
\text { (sans autre précision) }\end{array}$ & $\begin{array}{l}\text { Miel présumé d'oranger - Saveur de miel d'oranger - } \\
\text { Assez pauvre en pollen - Citrus, } 22 \text { - Olea, } 44 \text { - } \\
\text { Rosacées diverses, } 14 \text { - Echium, } 6 \text { - Papilionacées, } \\
4 \text { - Ombellifères, } 2 \text { - Composées, } 1 \text { - Divers et } \\
\text { indéterminés, } 7 . \\
\text { Présence de spores. }\end{array}$ \\
\hline
\end{tabular}

\section{TUNISIE}

\begin{tabular}{|c|c|c|c|c|}
\hline 84 & 3066 & 63 & Cap Bon & $\begin{array}{l}\text { Miel présumé d'oranger. } \\
\text { Citrus - Hedysarum - Echium - Ombellifères - Aca- } \\
\text { cia - Vitis - Oxalis. }\end{array}$ \\
\hline 85 & 3668 & 81 & Tunis-La Soukra & $\begin{array}{l}\text { Miel présumé d'oranger. } \\
\text { Citrus - Echium - Acacia - Lotus - Hedysarum - } \\
\text { Daucus - Eucalyptus - Olea - Rumex - Ombellifères - } \\
\text { Crucifères. }\end{array}$ \\
\hline 86 & 3669 & 81 & Tunis-La Soukra & $\begin{array}{l}\text { Miel présumé doranger - Récolté en } 1979 . \\
\text { Citrus - Acacia - Echium - Daucus - Lotus - Trifo- } \\
\text { lium - Cirsium. }\end{array}$ \\
\hline 87 & 3670 & 81 & Tunis-La Soukra & $\begin{array}{l}\text { Miel présumé d’oranger - Récolté en } 1980 . \\
\text { Citrus - Acacia - Echium - Hedysarum - Trifolium - } \\
\text { Cirsium - Ombellifères - Lotus. }\end{array}$ \\
\hline
\end{tabular}

Le pollen de Citrus est généralement sous-représenté dans les miels d'agrumes (MAURIZIO, 1949, 1958 ; VORWOHL, 1973) ; cependant, dans les échantillons $115 / 51$ et $467 / 53$ ce pollen dépasse les 50 pour cent, ce qui confirme les observations de VORWOHL (1973) sur la variabilité de la richesse en pollen des miels d'oranger.

Les pollens d'accompagnement des miels de Citrus sont sensiblement les mêmes que ceux des miels d'Eucalyptus. On notera cependant la proportion fréquemment plus élevée du pollen d'olivier (Olea) qui peut dépasser $20 \%$ 
et qui atteint $44 \%$ dans un miel marocain ayant par ailleurs toutes les caractéristiques d'un miel d'oranger $\left(\mathrm{N}^{\circ} 1545 / 62\right)$. Cette remarque nous conduit à préciser que si les oliviers ne sont pas considérés comme des plantes mellifères leurs fleurs sont cependant très visitées par les abeilles qui y récoltent un pollen abondant.

Une caractéristique essentielle des miels de Citrus est, d'autre part, la présence presque constante d'indices de miellat : spores de Fumagines, algues vertes microscopiques, fragments de mycélium. Signalons enfin la présence très fréquente de grains d'amidon.

\subsection{Miels de Romarin (Tabl. 4)}

Les miels de Romarin (Rosmarinus officinalis L.) typiques sont peu nombreux dans notre échantillonnage puisque seules l'Oranie et la Tunisie nous en ont fourni. Nous retrouvons là les données de l'étude de LAvIE (1976) qui va dans le même sens.

TABL. 4. - Pollen de Rosmarinus dominant

TABL. 4. - Dominant Rosmarinus pollen

\begin{tabular}{|c|c|c|c|c|}
\hline 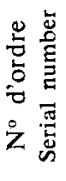 & 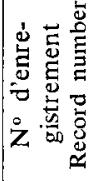 & 总总 & $\begin{array}{l}\text { Origine } \\
\text { des échantillons } \\
\text { Origin of samples }\end{array}$ & $\begin{array}{l}\text { Spectre pollinique - Observations } \\
\text { Pollen spectrum - Observations }\end{array}$ \\
\hline 21 & 3660 & 81 & $\begin{array}{c}\text { Algérie - Oranie } \\
\text { Tlemcen }\end{array}$ & $\begin{array}{l}\text { Miel de presse. } \\
\text { Rosmarinus, } 29 \text { - Eucalyptus, } 30 \text { - Sinapis, } 7 \text { - Om- } \\
\text { bellifères, } 11 \text { - Trifolium, } 4 \text { - Papaver, } 5 \text { - Divers, } 14 \\
\text { (Hedysarum - Chénopodiacées - Composées - Cistus - } \\
\text { Olea). }\end{array}$ \\
\hline 77 & 3057 & 68 & $\begin{array}{l}\text { Tunisie } \\
\text { Région de Kairouan } \\
\text { (Forêt de } \\
\text { la Kesra-Maktar) }\end{array}$ & $\begin{array}{l}\text { Miel présumé de Romarin - Miel d'extracteur. } \\
\text { Rosmarinus - Reseda - Olea - Eucalyptus - Cruci- } \\
\text { fères - Salix - Cistus - Erica - Papilionacées type } \\
\text { Ulex - Centaurea - Hedysarum. }\end{array}$ \\
\hline 79 & 3059 & 68 & $\begin{array}{l}\text { Tunisie - Région de } \\
\text { Kairouan (Sidi Bou } \\
\text { Baker - Pont du Fahs) }\end{array}$ & $\begin{array}{l}\text { Miel présumé de Romarin - Miel d'extracteur. } \\
\text { Rosmarinus - Cistus - Hedysarum - Salix - Cruci- } \\
\text { fères - Papilionacées type Ulex - Reseda - Erica - } \\
\text { Vicia. }\end{array}$ \\
\hline
\end{tabular}

Le miel algérien provient de la région de Tlemcen; le pollen de Romarin n'y est pas dominant ; le spectre pollinique est très diversifié. Il s'agit d'un miel de presse et c'est uniquement en fonction de notre expérience personnelle que nous avons jugé possible de le classer comme miel de Romarin (3660/81). Les deux 
miels tunisiens (3057/68 et 3059/68) proviennent de la région de Kairouan où le Romarin est abondant. Les spectres polliniques sont, dans ces deux échantillons, très diversifiés et représentatifs d'une végétation spontanée méditerranéenne.

\subsection{Miels de Trèfle (Tabl. 5)}

Quatre échantillons provenant de la région de Constantine (El Arrouch) ont été reçus entre 1953 et 1957. Ils sont caractérisés tous les quatre par l'abondance d'un pollen du type Trifolium repens associé à de nombreuses plantes secondaires parmi lesquelles on trouve de façon constante Eucalyptus, Hedysarum et Citrus.

\subsection{Miels de Sainfoin d'Espagne (Tabl. 5)}

Le Sainfoin d'Espagne ou Sulla, Hedysarum coronarium L. est une plante relativement commune dans une grande partie de l'Afrique du Nord. Les miels dans lesquels le pollen de cette plante est dominant ont été observés dans la partie centrale de l'Algérie, en Tunisie et au Maroc. Le pourcentage de pollen d'Hedysarum peut atteindre $90 \%$ ce qui est en rapport avec la présence de grandes surfaces cultivées consacrées à cette plante fourragère, notamment en Tunisie.

\subsection{Miels de Vipérine (Tabl. 5)}

Les miels dans lesquels domine le pollen de Vipérine (Echium sp.) sont tous originaires du Maroc et plus particulièrement de la partie nord du pays. Le pollen d'Echium peut atteindre $85 \%$. Il est associé à des pollens relativement variés parmi lesquels celui de Lavandula stoechas. La présence de miellat dans ces miels est assez constante.

\subsection{Divers pollens (Tabl. 5)}

Un miel de la région d'Alger (La Chiffa près de Blida) contient le pollen d'Olea à plus de $50 \%$.

Un échantillon de miel du Djebel Aures contient un pollen d'Artemisia à $95 \%$.

Provenant de Tebessa, un échantillon de miel contient un pollen d'Ombellifères non déterminé avec précision.

Enfin, de la région de Taroudant dans le Sud marocain, provient un échantillon contenant comme pollen dominant un pollen de Labiées non déterminé avec précision. 
TABL. 5. - Divers pollens dominants

TABL. 5. - Different dominant pollens

\begin{tabular}{|c|c|c|c|c|}
\hline 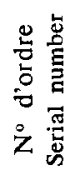 & 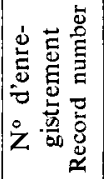 & 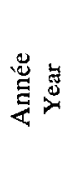 & $\begin{array}{c}\text { Origine } \\
\text { des échantillons } \\
\text { Origin of samples }\end{array}$ & $\begin{array}{c}\text { Spectre pollinique - Observations } \\
\text { Pollen spectrum - Observations }\end{array}$ \\
\hline
\end{tabular}

Type Trifolium repens

\begin{tabular}{l|l|l|l|l}
\hline 37 & 628 & 53 & $\begin{array}{l}\text { Est-Algérien } \\
\text { El Arrouch }\end{array}$ & $\begin{array}{l}\text { Trifolium sp., 51 - Eucalyptus, 23 - Crucifères, 1 - } \\
\text { Erica, 1 - Labiées, 2 - Ombellifères, 2 - Lotus, 2 - } \\
\text { Vicia, 3 - Citrus, 1 - Olea, 1 - Papilionacées, 6 - } \\
\text { Divers et indéterminés, 7. }\end{array}$ \\
\hline 39 & 732 & 54 & $\begin{array}{l}\text { Est-Algérien } \\
\text { El Arrouch }\end{array}$ & $\begin{array}{l}\text { Trifolium dominant - Isolés : Hedysarum - Euca- } \\
\text { lyptus - Citrus - Olea - Cistus. }\end{array}$ \\
\hline 40 & 904 & $\begin{array}{l}\text { Est-Algérien } \\
\text { El Arrouch }\end{array}$ & $\begin{array}{l}\text { Trifolium dominant. Isolés : Erica - Cistus - Ombel- } \\
\text { lifères - Citrus - Eucalyptus - Hedysarum. }\end{array}$ \\
\hline $\begin{array}{l}\text { Est-Algérien } \\
\text { El Arrouch }\end{array}$ & $\begin{array}{l}\text { Trifolium dominant - Isolés : Erica - Echium - } \\
\text { Ombellifères - Hedysarum - Borrago - Citrus - Olea - } \\
\text { Eucalyptus - Cistus - Borraginacées. }\end{array}$ \\
\hline
\end{tabular}

Hedysarum coronarium $\mathbf{L}$.

\begin{tabular}{|c|c|c|c|c|}
\hline 34 & 3667 & 81 & $\begin{array}{l}\text { Algérois } \\
\text { Azazga }\end{array}$ & $\begin{array}{l}\text { Hedysarum coronarium, } 90-\text { Erica - Cistus - Vicia - } \\
\text { Borraginacées. }\end{array}$ \\
\hline 72 & 707 & 54 & Maroc & $\begin{array}{l}\text { Hedysarum coronarium dominant - Accompagne- } \\
\text { ment : Lotus - Echium - Isolés : Lavandula stoechas - } \\
\text { Crucifères - Eucalyptus. } \\
\text { Miellat. }\end{array}$ \\
\hline 76 & 1543 & 62 & $\begin{array}{c}\text { Tunisie } \\
\text { La Marsa près Tunis }\end{array}$ & $\begin{array}{l}\text { Hedysarum coronarium, } 70-\text { Acacia, } 15 \text { - Citrus } 2 \text { - } \\
\text { Divers, } 3 .\end{array}$ \\
\hline 82 & 3062 & 68 & $\begin{array}{l}\text { Tunisie - Région de } \\
\text { Kairouan - Djebel } \\
\text { Ouseltia }\end{array}$ & $\begin{array}{l}\text { Hedysarum coronarium dominant - Isolés : Olea - } \\
\text { Crucifères - Helianthemum - Cistus - Rosmarinus - } \\
\text { Reseda - Taraxacum - Ceratonia - Marrubium - } \\
\text { Labiées diverses. }\end{array}$ \\
\hline
\end{tabular}

\section{Echium sp.}

\begin{tabular}{c|c|c|c|c}
\hline 60 & 103 & 51 & $\begin{array}{c}\text { Maroc } \\
\text { Région de Rharb }\end{array}$ & $\begin{array}{l}\text { Echium, 71-Eucalyptus, 10 - Trifolium, 1 - Helian- } \\
\text { themum, 4 - Labiées, 1 - Divers, 13. } \\
\text { Présence de miellat. }\end{array}$ \\
\hline 61 & -291 & -52 & $\begin{array}{c}\text { Maroc } \\
\text { Région de Rharb }\end{array}$ & $\begin{array}{l}\text { Echium, 45 - Trifolium, 6 - Eucalyptus, 5 - Rosacées } \\
\text { type Spiraea - Helianthemum - Nombreuses formes } \\
\text { indéterminées. } \\
\text { Présence de miellat. }\end{array}$
\end{tabular}


TABL. 5. - (suite)

\begin{tabular}{|c|c|c|c|c|}
\hline 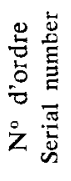 & 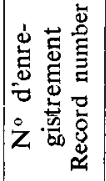 & 离 & $\begin{array}{c}\text { Origine } \\
\text { des échantillons } \\
\text { Origin of samples }\end{array}$ & $\begin{array}{l}\text { Spectre pollinique - Observations } \\
\text { Pollen spectrum - Observations }\end{array}$ \\
\hline 69 & 702 & 54 & Maroc & $\begin{array}{l}\text { Echium, } 69 \text { - Lavandula stoechas, } 8-\text { Cistus, } 2- \\
\text { Vicia, } 2 \text { - Papaver, } 2 \text { - Divers et indéterminés, } 17 . \\
\text { Présence de miellat. }\end{array}$ \\
\hline 70 & 705 & 54 & Maroc & $\begin{array}{l}\text { Echium, } 85 \text { - Lavandula stoechas } 5 \text { - Lotus, } 2 \text { - } \\
\text { Cistus, } 3 \text { - Plantago, } 1 \text { - Crucifère, } 1 \text { - Divers, } 3 . \\
\text { Présence de miellat. }\end{array}$ \\
\hline 71 & 706 & 54 & Maroc & $\begin{array}{l}\text { Echium, } 60 \text { - Lotus, } 25 \text { - Acacia, } 4 \text { - Ombellifères, } 2 \text { - } \\
\text { Eucalyptus, } 4 \text { - Olea, } 1 \text { - Papaver, } 1 \text { - Divers, } 3 . \\
\text { Présence de miellat. }\end{array}$ \\
\hline
\end{tabular}

Olea

\begin{tabular}{|c|c|c|c|c|}
\hline 23 & 634 & 53 & $\begin{array}{c}\text { Algérois } \\
\text { La Chiffa } \\
\text { près de Blida }\end{array}$ & $\begin{array}{l}\text { Présumé provenir du Geranium à parfum cultivé } \\
\text { Olea, } 52-\text { Echium, } 12 \text { - Composées, } 2 \text { - Crucifères, } \\
2 \text { - Labiées, } 2 \text { - Ombellifères, } 6 \text { - Papilionacées } \\
\text { diverses, } 7-\text { Trifolium, } 9 \text { - Rosacées, } 1 \text { - Citrus, } 1 \text { - } \\
\text { Divers, } 6 \text {. }\end{array}$ \\
\hline
\end{tabular}

Artemisia sp

\begin{tabular}{c|c|c|c|c}
\hline 42 & 3664 & 81 & $\begin{array}{c}\text { Est-Algérien } \\
\text { Djebel Aures }\end{array}$ & $\begin{array}{c}\text { Miel pressé - Artemisia sp., 95 - Hedysarum - Om- } \\
\text { bellifères - Divers. }\end{array}$ \\
\hline
\end{tabular}

Ombellifères

\begin{tabular}{c|c|c|c|l}
\hline 43 & 3665 & 81 & $\begin{array}{l}\text { Est-Algérien } \\
\text { Tebessa }\end{array}$ & $\begin{array}{l}\text { Ombellifères forme Ferula, 95 - Crucifères - Thy- } \\
\text { mus - Rosacées - Malvacées - Opuntia. } \\
\text { Présence de la masse finement granuleuse carac- } \\
\text { téristique des miels dombellifères. }\end{array}$ \\
\hline
\end{tabular}

Labiées.

\begin{tabular}{c|c|c|c|c}
\hline 73 & 910 & 57 & $\begin{array}{c}\text { Maroc } \\
\text { Taroudant }\end{array}$ & $\begin{array}{c}\text { Labiées forme Thymus - Isolé : Arctium - Nom- } \\
\text { breuses formes indéterminées. }\end{array}$ \\
\hline
\end{tabular}

\section{Miels dont le spectre pollinique ne comporte pas de pollen dominant}

Les miels dont le spectre pollinique ne comporte pas de dominante ont été classés en fonction de leur origine géographique (Tab. 6). 
TABL. 6. - Divers miels sans pollen dominant

TABL. 6. - Different honeys without dominant pollen

\begin{tabular}{|c|c|c|c|c|}
\hline 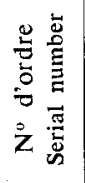 & 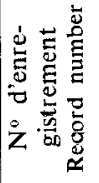 & 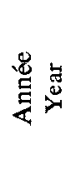 & $\begin{array}{c}\text { Origine } \\
\text { des échantillons } \\
\text { Origin of samples }\end{array}$ & $\begin{array}{c}\text { Spectre pollinique - Observations } \\
\text { Pollen spectrum - Observations }\end{array}$ \\
\hline
\end{tabular}

Miels de l'Oranie

\begin{tabular}{|c|c|c|c|c|}
\hline 4 & 113 & 51 & $\begin{array}{l}\text { Mohammadia } \\
\text { (ex Perrégaux) }\end{array}$ & $\begin{array}{l}\text { Echium, } 30 \text { - Forme Chenopodium, } 26 \text { - Borragi- } \\
\text { nacées type Symphytum, } 3 \text { - Crucifères, } 4 \text { - Erica, } 1 \text { - } \\
\text { Ombellifères, } 1 \text { - Papilionacées, } 2 \text { - Olea, } 1 \text { - Ro- } \\
\text { sacées, } 1 \text { - Papilionacée type Ulex, } 10 \text { - Eucalyptus, } \\
9 \text { - Citrus, } 1 \text { - Cistus, } 2 \text { - Divers, } 9 .\end{array}$ \\
\hline 5 & 114 & 51 & Mohammadia & $\begin{array}{l}\text { Présumé miel de «Thym kabyle»(?). Echium, } 25 \text { - } \\
\text { Composées, } 2 \text { - Centaurea, } 1 \text { - Crucifères, } 4 \text { - } \\
\text { Labiées, } 1 \text { - Ombellifères, } 7 \text { - Lotus, } 5 \text { - Trifolium, } \\
1 \text { - Citrus, } 4 \text { - Forme Ulex, } 6 \text { - Olea, 13 - Cistus, } 5 \text { - } \\
\text { Eucalyptus, } 5 \text { - Divers et indéterminés, } 21 .\end{array}$ \\
\hline 12 & 469 & 53 & Tlemcen - Beni-Saf & $\begin{array}{l}\text { Miel de montagne - Récolté au printemps. Labiée } \\
\text { type Rosmarinus, } 30 \text { - Echium, } 3 \text { - Composées, } 3 \text { - } \\
\text { Ombellifères, } 8 \text { - Papilionacées diverses, } 8 \text { - Rosacées } \\
\text { (Arbres fruitiers), } 5 \text { - Olea, } 16 \text { - Citrus, } 9 \text { - Salix, } 1 \text { - } \\
\text { Eucalyptus, } 1 \text { - Oxalis, } 3 \text { - Divers, 12. Miellat. }\end{array}$ \\
\hline 17 & 612 & 53 & Mohammadia & $\begin{array}{l}\text { Trifolium repens, } 30 \text { - Papilionacées diverses, } 24- \\
\text { Echium, } 7 \text { - Borraginacée, } 1 \text { - Crucifères, } 2 \text { - Eri- } \\
\text { ca, } 1 \text { - Ombellifères, } 13-\text { Vicia, } 2 \text { - Hedysarum, } 3 \text { - } \\
\text { Lotus, } 3 \text { - Citrus, } 3 \text { - Eucalyptus, } 3 \text { - Olea - Sedum, } \\
1 \text { - Divers, } 6 .\end{array}$ \\
\hline 18 & 632 & 53 & Mohammadia & $\begin{array}{l}\text { Présumé «miel d'oranger ». Olea, } 40 \text { - Citrus, } 20 \text { - } \\
\text { Graminées, } 1 \text { - Echium, } 1 \text { - Centaurea, } 2 \text { - Sinapis, } \\
2 \text { - Ombellifères, } 1 \text { - Papilionacées, } 3 \text { - Salix, } 1 \text { - } \\
\text { Eucalyptus, } 2 \text { - Ceratonia, } 12 \text { - Oxalis } 5 \text { - Divers, } 10 .\end{array}$ \\
\hline 22 & 3661 & 81 & $\begin{array}{l}\text { El-Bordj } \\
\text { (Mascara) }\end{array}$ & $\begin{array}{l}\text { Miel de montagne - Année de récolte } 1977 \text { - Pollens } \\
\text { d'accompagnement : Eucalyptus - Ceratonia - Pollens } \\
\text { isolés : Olea - Hedysarum - Vicia - Taraxacum - } \\
\text { Cistus - Helianthus - Asphodelus - Citrus - Palmiers - } \\
\text { Ombellifères. }\end{array}$ \\
\hline
\end{tabular}

Miels de l'Algérois

\begin{tabular}{|c|c|c|c|c|}
\hline 24 & 775 & 55 & $\begin{array}{l}\text { Khemis el Khechna } \\
\text { (environs d'Alger) }\end{array}$ & $\begin{array}{l}\text { Miel présumé Inula et Eucalyptus - Eucalyptus, } \\
65(*)-\text { Trifolium, } 17-\text { Citrus, } 2-\text { Olea, } 6 \text { - } \\
\text { Daucus, } 7 \text { - Crucifères, } 1 \text { - Arctium, } 1 \text { - Echium, } 1 . \\
\text { (Le pollen d'Inula n'a pas été observé). }\end{array}$ \\
\hline
\end{tabular}

(*) Ce miel n'a pas été considéré comme Eucalyptus le pollen de ces arbres étant toujours largement sur-représenté. 
TABL. 6. - (suite)

\begin{tabular}{|c|c|c|c|c|}
\hline 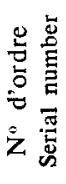 & 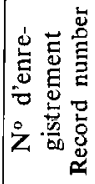 & 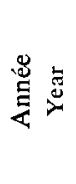 & $\begin{array}{l}\text { Origine } \\
\text { des échantillons } \\
\text { Origin of samples }\end{array}$ & $\begin{array}{c}\text { Spectre pollinique - Observations } \\
\text { Pollen spectrum - Observations }\end{array}$ \\
\hline 26 & 1630 & 63 & Boufarik & $\begin{array}{l}\text { Miel présumé d'oranger - Pollens d'accompagne- } \\
\text { ment : Trifolium - Hedysarum - Pollens isolés : } \\
\text { Citrus - Echium - Olea - Papaver - Composées Type } \\
\text { Leucanthemum. }\end{array}$ \\
\hline 29 & 3414 & 80 & Alger Hydra & $\begin{array}{l}\text { Miel récolté fin juin. Pollen d’accompagnement : } \\
\text { Eucalyptus - Pollens isolés : Acacia - Trifolium - } \\
\text { Taraxacum - Olea - Vitis - Trifolium - Cirsium - } \\
\text { Ombellifères. }\end{array}$ \\
\hline
\end{tabular}

Miels du Constantinois

\begin{tabular}{|c|c|c|c|c|}
\hline 36 & 290 & 52 & Daroussa & $\begin{array}{l}\text { Miel présumé d'oranger. } \\
\text { Echium, } 31 \text { - Cerinthe, } 10-\text { Borrago, } 2 \text { - Crucifères, } \\
1 \text { - Hedysarum, } 18-\text { Ulex, } 6 \text { - Salix, } 7 \text {, Papiliona- } \\
\text { cées, } 5 \text { - Eucalyptus, } 14-\text { Cistus, } 1 \text { - Acacia, } 2 \text { - } \\
\text { Divers, 3. }\end{array}$ \\
\hline 38 & 724 & 54 & El Arrouch & $\begin{array}{l}\text { Pollens daccompagnement. Trifolium - Daucus - } \\
\text { Pollens isolés : Papilionacées - Eucalyptus - Citrus. }\end{array}$ \\
\hline 44 & 3666 & 81 & $\begin{array}{l}\text { Constantine } \\
\text { (environs de) }\end{array}$ & $\begin{array}{l}\text { Pollens d’accompagnement : Eucalyptus - Pollens } \\
\text { isolés : Hedysarum - Citrus - Erica - Trifolium - } \\
\text { Lavandula stoechas - Ombellifères - Chénopodiacée - } \\
\text { Mentha - Cistus - Cirsium - Arctium. }\end{array}$ \\
\hline 45 & 3754 & 82 & Tebessa & $\begin{array}{l}\text { Papilionacées - Rosacées - Ombellifères - Crucifères - } \\
\text { Convolvulus - Centaurea - Cirsium - Nombreuses } \\
\text { formes non identifiées. } \\
\text { Nombreux indices de miellat. }\end{array}$ \\
\hline 46 & 3756 & 82 & $\begin{array}{c}\text { Région } \\
\text { de Constantine }\end{array}$ & $\begin{array}{l}\text { Miel présumé de Chardon («Guernina»). Type Cir- } \\
\text { sium - Eucalyptus - Rosacées type Rubus - Trifo- } \\
\text { lium - Echium - Labiées type Rosmarinus - Cistus - } \\
\text { Hedysarum-Erica. }\end{array}$ \\
\hline 47 & 3757 & 82 & $\begin{array}{l}\text { Région } \\
\text { de Constantine }\end{array}$ & $\begin{array}{l}\text { Miel présumé d'oranger. Pauvre en pollen. Hedy- } \\
\text { sarum - Circium - Borrago - Erica - Citrus. } \\
\text { Le pollen de Citrus est à l'état de pollen isolé. }\end{array}$ \\
\hline 48 & 3758 & 82 & $\begin{array}{l}\text { Région } \\
\text { de Constantine }\end{array}$ & $\begin{array}{l}\text { Miel présumé de Sainfoin. Onobrychis - Asphodelus - } \\
\text { Hedysarum - Cistus - Crucifères. } \\
\text { Miel pauvre en pollen. }\end{array}$ \\
\hline 49 & 3759 & 82 & $\begin{array}{l}\text { Région } \\
\text { de Constantine }\end{array}$ & $\begin{array}{l}\text { Miel présumé de Capsella et Raphanus - Crucifères - } \\
\text { Asphodelus - Eucalyptus - Papilionacées type Ulex - } \\
\text { Hedysarum - Lotus - Onobrychis. }\end{array}$ \\
\hline
\end{tabular}


TABL. 6. - (suite)

\begin{tabular}{|c|c|c|c|c|}
\hline 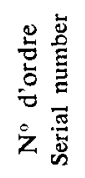 & 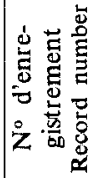 & 兽 & $\begin{array}{l}\text { Origine } \\
\text { des échantillons } \\
\text { Origin of samples }\end{array}$ & $\begin{array}{c}\text { Spectre pollinique - Observations } \\
\text { Pollen spectrum - Observations }\end{array}$ \\
\hline
\end{tabular}

\begin{tabular}{c|c|c|c|c|c}
50 & 3760 & 82 & $\begin{array}{l}\text { Région } \\
\text { de Constantine }\end{array}$ & $\begin{array}{l}\text { Miel présumé de Peganum harmala (Zygophylla- } \\
\text { cées) - Présence d'un pollen dominant de Zygophyl- } \\
\text { lacées en cours d'identification. }\end{array}$ \\
\hline 51 & 3761 & 82 & $\begin{array}{l}\text { Région } \\
\text { de Constantine }\end{array}$ & $\begin{array}{l}\text { Miel présumé de Cirsium. Echium - Asphodelus - } \\
\text { Crucifères. } \\
\text { Pas de pollen de Cirsium. }\end{array}$ \\
\hline 53 & 3763 & 82 & Région de Constantine & Erica - Lavandula - Eucalyptus. \\
\hline
\end{tabular}

Miels du Sud-Algérien

\begin{tabular}{c|c|c|c|l}
\hline 54 & 3662 & 81 & $\begin{array}{l}\text { Laghouat } \\
\text { (Sahara) }\end{array}$ & $\begin{array}{l}\text { Spectre pollinique complexe comportant de nom- } \\
\text { breuses formes non identifiées - En outre : Euca- } \\
\text { lyptus - Plusieurs formes d'Ombellifères - Hedysarum. }\end{array}$ \\
\hline 55 & 3663 & 81 & $\begin{array}{l}\text { Laghouat } \\
\text { (Sahara) }\end{array}$ & $\begin{array}{l}\text { Spectre pollinique complexe comportant de nom- } \\
\text { breuses inconnues. En outre : Eucalyptus - Arctium - } \\
\text { Trifolium - Ombellifères. } \\
\text { Il s'agit d'un miel de presse. }\end{array}$ \\
\hline
\end{tabular}

Miels du Maroc

\begin{tabular}{|c|c|c|c|c|}
\hline 65 & 292 & 52 & $\begin{array}{ll}\text { Région } \\
\text { de Casablanca }\end{array}$ & $\begin{array}{l}\text { Miel présumé d'oranger et lavande. Rosacée type } \\
\text { Spiraea - Echium - Labiées diverses - Lavandula } \\
\text { stoechas - Ombellifère - Eucalyptus - Cistus - Cha- } \\
\text { maerops. Présence de miellat. }\end{array}$ \\
\hline 66 & 293 & 52 & $\begin{array}{l}\text { Région } \\
\text { de Tanger }\end{array}$ & $\begin{array}{l}\text { Miel présumé de lavande et vipérine. Echium, } 40 \text { - } \\
\text { Rosacée type Spiraea, } 30 \text { - Lavandula stoechas, } 4 \text { - } \\
\text { Acacia, } 1 \text { - Cistus, } 19-\text { Eucalyptus, } 1 \text { - Erica, } 1 \text { - } \\
\text { Divers, } 4 . \\
\text { Présence de miellat. }\end{array}$ \\
\hline 67 & 525 & 53 & Maroc & $\begin{array}{l}\text { Lavandula stoechas, 27, Echium, } 25 \text { - Crucifères, } 8 \text { - } \\
\text { Ombellifères, } 6 \text { - Vicia, } 3 \text { - Acacia, } 1 \text { - Rosacées, } 1 \text { - } \\
\text { Cistus } 4 \text { - Eucalyptus, } 2 \text { - Centaurea, } 1 \text { - Composées, } \\
2 \text { - Papilionacées, } 3 \text { - Divers, } 17 .\end{array}$ \\
\hline 68 & 545 & 53 & Maroc & $\begin{array}{l}\text { Miel présumé de Lavande. Echium - Eucalyptus - } \\
\text { Rosacées type Spiraea - Lotus - Papilionacées forme } \\
\text { Ulex - Helianthemum - Lavandula. } \\
\text { Présence de miellat. }\end{array}$ \\
\hline 74 & 1123 & 58 & Maroc & $\begin{array}{l}\text { Eucalyptus - Echium - Hedysarum - Trifolium - } \\
\text { Acacia. } \\
\text { Présence de miellat. }\end{array}$ \\
\hline
\end{tabular}


TABL. 5. - (suite)

\begin{tabular}{|c|c|c|c|c|}
\hline 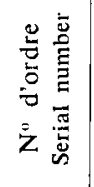 & 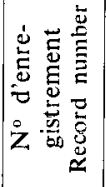 & 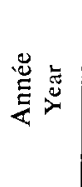 & $\begin{array}{c}\text { Origine } \\
\text { des échantillons } \\
\text { Oriyin of samples }\end{array}$ & $\begin{array}{c}\text { Spectre pollinique - Observations } \\
\text { Pollen spectrum - Observations }\end{array}$ \\
\hline
\end{tabular}

Miels de Tunisie

\begin{tabular}{|c|c|c|c|c|}
\hline 78 & 3058 & 68 & $\begin{array}{l}\text { Menzel Temine } \\
\text { (Cap Bon) }\end{array}$ & $\begin{array}{l}\text { Miel de presse - Présumé Eucalyptus-oranger. Hedy- } \\
\text { sarum - Amygdalus - Citrus - Vicia - Diplotaxis - } \\
\text { Echium - Convolvulus - Graminée - Acacia - Euca- } \\
\text { lyptus - Sinapis - Arbres fruitiers - Rumex - Papa- } \\
\text { ver - Olea - Ombellifères - Helianthus - Medicago - } \\
\text { Borraginacées - Lotus - Divers. }\end{array}$ \\
\hline 81 & 3061 & 68 & $\begin{array}{l}\text { Menzel Bou Zelfa } \\
\text { (Cap Bon) }\end{array}$ & $\begin{array}{l}\text { Miel présumé d’oranger et fleurs diverses. Citrus } \\
\text { Rosmarinus - Eucalyptus - Ombellifères - Hedy- } \\
\text { sarum - Diplotaxis - Echium - Citrus - Arbres } \\
\text { fruitiers - Thymus - Olea - Convolvulus - Papaver - } \\
\text { Acacia - Composées diverses - Présence de miellat. }\end{array}$ \\
\hline 83 & 3063 & 68 & Sedjenane & $\begin{array}{l}\text { Miel présumé d'Eucalyptus. Contient de l'amidon et } \\
\text { des impuretés diverses. } \\
\text { Eucalyptus - Lavandula stoechas - Olea - Hedysa- } \\
\text { rum - Cirsium. }\end{array}$ \\
\hline
\end{tabular}

\subsection{Miels de l'Oranie}

Sur les 22 miels de l'Oranie on remarque que 6 seulement d'entre eux sont des miels dont le spectre pollinique ne comporte pas de dominance. Cependant on y retrouve secondairement les pollens d'Eucalyptus et de Citrus. Les spectres polliniques sont complexes avec présence assez constante d'Olea, Echium, Crucifères, Ombellifères.

\subsection{Miels de l'Algérois}

Les miels de l'Algérois sont, en majorité, des miels où domine le pollen d'Eucalyptus. Il n'y a que trois échantillons sans dominance mais dans lesquels on retrouve sensiblement la même flore que dans les miels de l'Oranie.

\subsection{Miels du Constantinois}

Sur les 17 miels provenant du Constantinois 10 sont sans dominance pollinique. On note un contraste assez net entre les miels de la période ancienne et les plus récents. Ce contraste n'est pas forcément l'indice d'une évolution de l'agriculture ; en fait, il s'agit de miels qui, bien que produits dans la même région géographique 
sont issus de milieux très différents. Le spectre des miels de la période actuelle comporte une proportion relativement élevée de pollens dont l'identification est encore imprécise.

\subsection{Miels du Sud algérien}

Les deux miels provenant de Laghouat ont un spectre pollinique particulièrement complexe et dont une partie seulement a pu faire l'objet d'identifications relativement précises.

\subsection{Miels du Maroc}

Dans les miels marocains sans dominance pollinique on remarque que les pollens d'Echium et de Lavandula stoechas sont relativement constants. On note également que les indices de miellat sont plus fréquents que dans les autres échantillons.

\subsection{Miels de Tunisie}

Les trois miels de Tunisie sans dominance sont remarquables par l'abondance des taxons représentés mais l'échantillon provenant de Sedjenane contient trop d'amidon d'origine inconnue pour que la préparation microscopique soit entièrement lisible.

\section{DISCUSSION}

L'ensemble des trois pays du Maghreb représente un immense territoire comportant des zones climatiques contrastées auxquelles correspondent des zones de végétation très diversifiées. Les colonies d'abeilles y trouvent donc des conditions de vie fort différentes selon qu'elles sont dans les régions côtières, dans les plaines cultivées, dans les montagnes ou en bordure du désert. La flore dont elles tirent leur subsistance est très hétérogène et pour en connaître tous les aspects il serait nécessaire de procéder à une étude systématique basée à la fois sur des études botaniques réalisées sur le terrain ainsi que sur un plan de prélèvement d'échantillons de miel permettant d'obtenir une image fidèle et relativement complète des ressources mellifères des différents milieux. L'étude dont nous présentons ici les résultats ne répond pas à ces conditions. Elle a été réalisée de l'extérieur et au hasard des envois spontanés d'échantillons de miel qui s'échelonnent sur une longue période et qui ne couvrent qu'une portion du territoire maghrébin. On ne peut donc en attendre que des conclusions partielles dont le seul intérêt est d'apporter quelques connaissances nouvelles sur les miels d'un très vaste secteur géographique jusqu'ici peu étudié du point de vue mélisso-palynologique. 
L'abondance et la relative homogénéité des miels dont le spectre comporte les Eucalyptus comme pollens dominants montre que nous sommes en présence d'une ressource mellifère importante, principalement dans l'Algérois et l'Oranie. Il ne faut cependant pas perdre de vue que les miels d'Eucalyptus sont en général riches en pollen (Maurizio, 1949 ; Barbier et Pangaud, 1961) et que les spectres où le pollen d'Eucalyptus est représenté à près de $100 \%$ doivent être considérés de façon critique. Seule une étude approfondie à la fois des espèces représentées et des caractères physico-chimiques et organoleptiques des miels d'Eucalyptus du Maghreb permettra d'évaluer de façon correcte l'importance apicole réelle de ces arbres. L'omniprésence du pollen d'Eucalyptus dans les miels d'Afrique du Nord est donc confirmée mais sa signification profonde reste à établir de façon scientifique.

Les miels communément appelés «miels d'oranger» sont fréquents aussi bien en Algérie qu'en Tunisie et au Maroc. Les échantillons algériens dont nous disposons sont anciens. Seuls les miels d'oranger de Tunisie sont récents mais trop peu nombreux pour qu'on puisse en faire une étude approfondie. La question des miels d'oranger du Maghreb est à reprendre entièrement pour tenir compte des conditions actuelles de production. L'évolution de la composition variétale des vergers d'agrumes et l'effet dissuasif des traitements phytosanitaires sur l'exploitation de la miellée d'orangers par les apiculteurs sont des éléments qu'il faudrait prendre à présent en considération.

Une analyse détaillée de nos résultats et leur regroupement en tableaux synthétiques alourdirait inutilement cette étude sans apporter d'éléments d'importance décisive. Seules des études ponctuelles conduites de façon systématique pourront aboutir à des déterminations dépassant le stade du genre pour atteindre celui de l'espèce. Il paraît dès à présent évident qu'un effort devrait être fait au niveau des Ombellifères, difficiles à déterminer, mais qui semblent jouer en Afrique du Nord un rôle important. De même en ce qui concerne les Papilionacées, les Crucifères et les Borraginacées pour lesquelles des déterminations au niveau de l'espèce seraient très utiles.

Mais, dès à présent, on peut considérer que les miels d'Afrique du Nord actuellement connus se distinguent assez bien des miels du reste du Bassin méditerranéen. Le rôle important joué par les Eucalyptus, les Ombellifères et les Papilionacées et, d'autre part, la faible représentation des Ericacées, Cistacées et même Labiées montre l'hétérogénéité des miels du Bassin méditerranéen. Il est certain que les études dont on dispose (OustuanI, 1976; Ricciardelli D'Albore, 1980 ; Vorwohl, 1973 ; Louveaux, 1973) pour les autres parties de ce Bassin font apparaître une flore mellifère nettement différente.

En conclusion, on peut dire que l'étude des miels d'Afrique du Nord est à peine ébauchée. Si elle doit se poursuivre elle devra se faire sur place et avec 
une méthodologie nouvelle associant aux études polliniques qualitatives et quantitatives les études physico-chimiques indispensables à une connaissance effective des ressources mellifères de cette vaste région.

Reçu pour publication en avril 1984.

\section{REMERCIEMENTS}

Cette étude n'a été possible que parce que, pendant plus de 30 ans, nos amis apiculteurs d'Afrique du Nord nous ont envoyé pour notre documentation des échantillons de miels de la production locale. Ne pouvant tous les citer nous leur adressons des remerciements collectifs. Nous avons, malheureusement, perdu la trace de beaucoup d'entre eux. Parmi ceux qui nous le plus aidé nous tenons à citer $M$. Camille Paradeau et $M$. Jean-Claude Laurent à qui nous devons bon nombre d'échantillons de l'Oranie et du Constantinois.

\section{ZUSAMMENFASSUNG}

\section{NORDAFRIKANISCHE HONIGE UND IHR POLLENSPEKTRUM}

Es gibt immer noch eine breite Kluft zwischen der Kenntnis der europäischen Honige und den Honigen aus anderen Teilen der Welt. Die Honige des westlichen mediterranen Beckens und insbesondere die von Nordafrika sind in bezug auf ihr Pollenspektrum immer noch relativ unbekannt. Das ist besonders deshalb bedauernswert, da es sich um ein großes Gebiet mit möglicherweise reichem Angebot an Honig liefernden Pflanzen und gutem Klima für Honigbienen handelt.

Zwischen 1949 und 1982 hat die Station de Recherches sur l'Abeille et les Insectes Sociaux eine große Anzahl an Honigproben gesammelt. 87 Proben (16 aus Marokko, 59 aus Algerien und 12 aus Tunesien) wurden nach der Standardmethode der Melissopalynologie (Louveaux, Maurizio und VORWOHL, 1978) analysiert.

Die Resultate sind in 6 Tabellen aufgelistet. Tab. 1 gibt eine Liste der Proben mit Registriernummer und -datum, sowie geographischer und botanischer Herkunft. Die Tabellen 2-5 geben die Resultate der Einzelanalysen (Probe für Probe) wieder. Jeder Honig wurde nach seinem dominanten Pollen klassifiziert. In Tab. 6 sind Honigproben verzeichnet, deren Spektrum keinen dominanten Pollen aufwies.

Eukalyptus-Pollen kommt in den Honigen Nord-Afrikas sehr häufig vor, ganz besonders in Algerien. Honige mit fast $100 \%$ Eukalyptus Pollen waren nicht selten. Der sogenannte Orangenblüten-Honig kam vor allem aus Oranie (Algerien) und Tunesien. Sein Gehalt an Citrus-Pollen ist variabel, wie auch von MaUrizio (1958) und VorwoHL (1973) beschrieben. Rosmarin-Honig wurde in Nord-Afrika selten gefunden. Er kommt in wenigen Gegenden vor, wie von Lavie (1976) berichtet.

Das Pollenspektrum der Honige aus dem Maghreb unterscheidet sich stark von dem des restlichen mediterranen Beckens, jedoch werden noch weitere Analysen zur Bestimmung der sekundären und untergeordneten Pollen auf dem Artniveau gebraucht. Pollen von Umbelliferen und Papilionaceen bedürfen tiefergehender Untersuchungen. 


\section{SUMMARY}

\section{NORTH AFRICAN HONEYS AND THEIR POLLEN SPECTRUM}

There is still a large gap between our knowledge of European honeys and that of honeys from other parts of the world. Honeys from the western part of the Mediterranean Basin, and in particular from North Africa, remain relatively unknown with respect to their pollen spectrums. This is unfortunate as it concerns a large area which is potentially rich in honey plants and has a climate favorable for honeybees.

Between 1949 and 1982 the Station de Recherches sur l'Abeille et les Insectes sociaux collected a large number of samples of honey. Eighty-seven samples (16 from Morocco, 59 from Algeria and 12 from Tunisia) were analysed for pollen following the standard methods of melissopalynology (J. Louveaux, A. Maurizio and G. Vorwohl, 1978).

Results are presented in 6 tables. The first table gives the list of samples classified according to the date, geographical and botanical origin. Tables 2-5 give results of analyses, sample by sample. Each honey is classified according to its dominant pollen. Table 6 shows samples in which the spectrum showed no dominant pollen.

Eucalyptus pollen is very frequent in the spectrum of honeys from North Africa and more particularly from the Algerois. Honeys with nearly $100 \%$ Eucalyptus pollen are not rare. The so-called orange-blossom honeys occur principally in Oranie and Tunisia. Their content of citrus pollen is variable confirming the bibliographic data (A. MAUrizio, 1958, G. VorwoHL, 1973). Rosemary honeys are rare in North Africa. They do occur in some areas as reported by P. LAVIE (1976).

Large differences appear between the pollen spectrums of honeys from the Maghreb and that from the remaining Mediterranean Basin, but further studies relating to the determination of secondary and minor pollens at the species level are still needed. Pollens from Umbelliferae and Papilionaceae would require more detailed studies.

\section{BIBLIOGRAPHIE}

Barbier E., Pangaud C.Y., 1961. - Origine botanique et caractéristiques physico-chimiques des miels. Ann. Abeille, 4 (1), 51-65.

Feller-Demalsy M.J., 1983. - Le spectre pollinique des miels du Québec. Apidologie, 14 (3), 147-174.

Fossel A., Ruttner F., 1966. - Die kleine Wachsblume, Cerinthe minor L., eine characteristische Trachtpflanze pannonischer und alpiner Trockengebiete. Z. Bienenforsch., 8, 168-177.

LAviE P., 1971. - Le Romarin, Rosmarinus officinalis L. Bull. tech. apic., 3 (1), 15-26.

Louveaux J., 1968. - L'analyse pollinique des miels, in Chauvin R., Traité de Biologie de l'abeille, Tome 3, 325-362, Masson ed. Paris.

Louveaux J. 1973. - Les miels de Romarin (Rosmarinus officinalis L.). Apidologie, 4 (2) 166.

Louveaux J., Maurizio A., Vorwohl G., 1978. - Methods of Mellissopalynology. Bee World, 59 (4), 139-157.

Maurizio A., 1949 - Pollenanalytische Untersuchungen an Honig und Pollenhöschen. Beih. Schweiz. Bienenztg., 2 (18), 320-421.

Maurizio A., 1958. - Beiträge zur quantitativen Pollenanalyse des Honigs. 3. Absoluter Gehalt pflanzlicher Bestandteile in Esparsettte, Luzerne, Orangen und Rapshonigen. Ann. Abeille, 1 (2), 93-106. 
MaUrizro A., 1960. - Das mikroskopische Bild jugoslawischer Importhonige. Z. Bienenforschung, 5 (1), 8-22.

Maurizio A., 1975. - Microscopy of Honey. In E. Crane ed., Honey : a comprehensive survey, p. 240-257, London, Heinemann.

Maurizio A., Louveaux J., 1965. - Pollen de plantes mellifères d'Europe. — Paris, Union des Groupements Apicoles Français, 148 p.

Oustuani A.M., 1976. - Das mikroskopische Bild der Honige des östlichen Mittelmeergebietes. Diss. Hohenheim, $62 \mathrm{p}$.

Paradeau C., 1978. - L'apiculture en Algérie. Rev. fr. apicult., Nº 363, 185-186.

Pfister R., 1895. - Versuch einer Mikroskopie des Honigs. Forschungsber. Lebensmitt, u. ihre Bez. z. Hygiene for. Chem. Pharm. München, 2, 29.

Ricciardell D'Albore G., 1980. - Contributo alla conoscenza della flora nettarifera del Marocco sulla base dell'analisi microscopica dei mieli. Riv. Agric. Subtrop. Trop., 74 (1-2) 59-71.

Ricciardelli D'Albore G., Vorwohl G., 1980. - Sortenhonige im Mittelmeergebiet. Riv. Agric. Subtrop. Trop., 74 (1-2), 89-118.

RutTNer F. 1956. - Oberösterreichische Honige. Bienenvater, 77 (3), 82-90.

Ruttner F., 1961. - Der Pollen der Eichenmistel (Loranthus europaeus Jacq.) als Charakterform in österreichischen Honigen. Z. Bienenforsch., 5 (7), 220-226.

Ruttner F., 1964. - Zur pollenanalytischen Diagnose südosteuropäischer Honige. Ann. Abeille, 7 (4), 321-327.

SKENDER K., 1972. - Situation actuelle de l'apiculture algérienne et ses possibilités de développement. I.N.A. Alger, Mémoire d'ingéniorat.

VorwoHL G., 1973. - Die Repräsentierung des Citrus-Pollens in italienischen Orangenhonigen. Apidologie, 4 (3), 275-281.

Vorwohl G., 1973. - Das Pollenbild der tunesischen Honige. Apidologie, 4 (2), 168.

Vorwohl G., 1981. - Pollen spectra of African honeys, IV Int. Palynol. Conf. Lucknow (1976-1977) 3, 499-502.

ZANDER E. - Beiträge zur Herkunftsbestimmung bei Honig. Vol. I, Berlin, Reichsfachgruppe, 1935; vol. II et III, Leipzig Liedloff, Loth \& Michaelis, 1937 et 1941; vol. IV, München, F. Ehrenwirth, 1949 ; vol. V Leipzig, Liedloff, Loth \& Michaelis, 1951. 